\title{
Unmet needs of child and adolescent psychiatrists among Asian and European countries: does the Human Development Index (HDI) count?
}

\author{
Andre Sourander ${ }^{1} \cdot$ Roshan Chudal $^{1} \cdot$ Norbert Skokauskas $^{2} \cdot$ Ahmed Malallah Al-Ansari $^{3} \cdot$ Anat Brunstein Klomek $^{4}$. \\ Chanvit Pornnoppadol ${ }^{5} \cdot$ Gerasimos Kolaitis $^{6}$. Junko Maezono ${ }^{1} \cdot$ Hans-Cristoph Steinhausen ${ }^{7,8,9}$. \\ Helena Slobodskaya ${ }^{10} \cdot$ Hitoshi Kaneko $^{11}$. Jaya Regmee ${ }^{12}$. Liping Li ${ }^{13} \cdot$ Mai Huong Nguyen ${ }^{14} \cdot$ Meytal Grimland $^{15}$. \\ Olga Osokina ${ }^{16}$. Say How Ong ${ }^{17}$. Samir Kumar Praharaj ${ }^{18}$. Sigita Lesinskienè ${ }^{19}$. Sturla Fossum ${ }^{20}$. Tjhin Wiguna ${ }^{21}$. \\ Valentina A. Makasheva ${ }^{22} \cdot$ Venla Lehti ${ }^{1,23}$
}

Published online: 29 December 2017

(c) Springer-Verlag GmbH Germany, part of Springer Nature 2017

In the Western world, it is estimated that between one-third to one-fourth of children and adolescents meet the diagnostic criteria for a mental disorder in their lifetime [1]. More recently, a meta-analysis of studies from 27 countries reported the worldwide-pooled prevalence of mental disorders of $13.4 \%$ and the variability in prevalence estimates were not explained by geographic location [2]. More than half of mental health disorders in adulthood start by the age of 14 years [3]. However, there are various limitations for

Andre Sourander and Roshan Chudal shared first authorship.

Andre Sourander

andsou@utu.fi

1 University of Turku and Turku University Hospital, Turku, Finland

2 Norwegian University of Science and Technology, Trondheim, Norway

3 Arabian Gulf University, Manama, Bahrain

4 Interdisciplinary Center (IDC) Herzliya, Herzliya, Israel

5 Mahidol University, Bangkok, Thailand

6 School of Medicine, National and Kapodistrian University of Athens, Athens, Greece

7 University of Zurich, Zürich, Switzerland

8 University of Basel, Basel, Switzerland

9 Capital Region Psychiatry, Copenhagen, Denmark

10 Institute of Physiology and Basic Medicine, Russia Novosibirsk State University, Novosibirsk, Russia

11 Nagoya University, Nagoya, Japan

12 Child and Adolescent Psychiatry Department, Kanti Children's Hospital/Child Workers in Nepal Concerned Centre (CWIN), Kathmandu, Nepal obtaining correct figures. Many child and adolescent mental health (CAMH) surveys start with an inclusion cut-off age of 15 or 25 years, there are concerns about privacy limiting the collection of sensitive information from young children, and school surveys miss out on the population that are out of school, more so among low- and middle-income countries [4]. Thus, an early disease onset, chronic disease course, coupled with possible underestimation of the disease estimates highlights the enormity of the global burden of $\mathrm{CAMH}$ problems. While there has been growing awareness

13 Shantou University and Medical School, Shantou, China

14 Department of Psychiatry, National Hospital of Pediatrics, Hanoi, Vietnam

15 Tel Aviv University, Tel Aviv, Israel

16 Donetsk National Medical University, Donetsk, Ukraine

17 Institute of Mental Health, Singapore, Singapore

18 Department of Psychiatry, Kasturba Medical College, Manipal, Manipal University, Manipal, India

19 Faculty of Medicine, Vilnius University, Vilnius, Lithuania

20 University of Troms $\emptyset$, Troms $\varnothing$, Norway

21 Dr. Cipto Mangukusumo General Hospital-Faculty of Medicine Universitas of Indonesia, Jakarta, Indonesia

22 Novosibirsk Regional Psycho-neurology Clinic for Children and Adolescents, Novosibirsk, Russia

23 Department of Psychiatry, Helsinki University Hospital and University of Helsinki, Helsinki, Finland 
of the burden of CAMH in high-income countries, there are huge unmet needs in low-income and middle-income countries [5, 6]. Moreover, recent estimates of the numbers of professionals working in child and adolescent psychiatry (CAP) have been lacking, as the last available World Health Organization (WHO) Child Mental Health Atlas is more than a decade old [5].

The Eurasian Child Mental Health Study (EACMHS), established September 2016, includes CAMH experts in ten Asian and seven European countries: Bahrain, China, Finland, Greece, India, Indonesia, Israel, Japan, Lithuania, Nepal, Norway, Russia, Singapore, Switzerland, Thailand, Ukraine and Vietnam [7, 8]. The overall aim of the group is to conduct cross-cultural, multi-site research on the wellbeing and mental health of children and adolescents.

This editorial reports the ratios of child and adolescent psychiatrists per population size of 100,000 children aged 14 years or younger. The age limit of 14 years was made to ensure a uniform data source for children's population as the World Bank data provide information on children aged 14 or younger in these countries [9]. The countries were arranged according to their ranking in the Human Development Index (HDI) 2016, put together by the United Nations Development Programme (UNDP). The HDI summarizes key dimensions of human development, including having a long and healthy life, being knowledgeable and having a decent standard of living [10]. The countries included in the HDI list are sub-grouped into four categories in the declining order of the HDI rank: (1) very high human development (Rank 1-51), (2) high human development (52-105), (3) medium human development (107-147) and (4) low human development (148-188). Information about the numbers of child and adolescent psychiatrists and training programs (updated February 2017) was obtained from group members using a structured questionnaire (available on request).

Overall, the ratio of child and adolescent psychiatrists for 100,000 children aged 14.0 years or younger ranged from 0.01 in Nepal to 48.3 in Switzerland (Fig. 1). The three European countries with the highest HDI rankings also had the highest ratios of child and adolescent psychiatrists: Norway (ranking 1, ratio 47.74), Switzerland $(3,48.30)$ and Finland $(23,45.40)$. The rankings and ratios among the other four European countries were Greece (29, 22.24), Lithuania $(37,13.77)$, Russia $(49,5.31)$ and Ukraine $(84,4.34)$. The Asian countries with the highest HDI rank also had the highest CAP ratios in the region: Singapore $(5,3.95)$, Japan $(17,1.97)$ and Israel $(19,11.29)$. The ranks and ratios among other high HDI ranked Asian countries were: Bahrain (47, 2.07), Thailand $(87,1.57)$ and China $(90,0.09)$. The ranks and ratios among medium HDI ranked countries were: Indonesia $(113,0.07)$, India $(131,0.02)$ and Nepal $(144,0.01)$ There were no child and adolescent psychiatrists in Vietnam $(115,0)$.
There was a wide variation in CAP training practices among the countries. A specialized postgraduate training in CAP was non-existent in only few countries (Vietnam, Nepal). Among other countries, it varied between 3 months (Ukraine) and 72 months (Finland, Switzerland). While some countries had no inclusion of CAP during general psychiatry training (China, Finland), the duration among other countries ranged from 1 month (Ukraine) to 12 months (Israel, Norway and Nepal). There have also been some positive developments in CAP training as countries like India have recently started specialized training programs in CAP. In addition, since 2017, a 3-month CAP course has been started in Vietnam for general psychiatrists and clinical psychologists working with children and adolescents.

In the European countries, the child and adolescent psychiatrists ratio declined as the HDI fell. Although a similar trend was also seen among the Asian countries, an important observation was that several very high HDI ranked Asian countries had much lower ratios than European countries with similar ranks. For example, in the three European countries with the highest HDI, there was more than one child and adolescent psychiatrist for every 2200 children up to the age of 14, while in Singapore and Japan, two Asian countries with a very high HDI and high-quality healthcare, there was only one child psychiatrist for every 26,000 and 56,000 children, respectively, of that age. One of the most notable and enormous discrepancies in the global availability of child psychiatrists was that in two of the world's most populated countries, China and India, there were only one or two child psychiatrists for over one million children.

CAP specialization currently lacks an international standardized training program. This lack of uniformity in the knowledge base, practice skills unique to the field, and failure to timely adapt to the changing scenario of the science and practice of CAP has hampered achieving the best possible results [11]. There have been some efforts at the regional level to address these challenges with the European Union of Medical Specialists Section on Child and Adolescent Psychiatry (UEMS-CAP) and The European Federation of Psychiatric Trainees (EFPT) working in the direction of creating a common European standard in CAP training [12, 13]. These efforts could be used as a framework for development of training programs in CAP in other countries.

There are a few limitations of this study that need to be considered. First, the discrepancy in the ratio of child and adolescent psychiatrists could be due to the fact that the specialized training programs in CAP are non-existent or have only recently started in several countries. It is likely that in these countries, a significant proportion of mental health needs of children and adolescents are addressed by general psychiatrists, pediatricians and general practitioners. Second, differences in the reimbursement for health care, either through the availability of national health insurance 
a

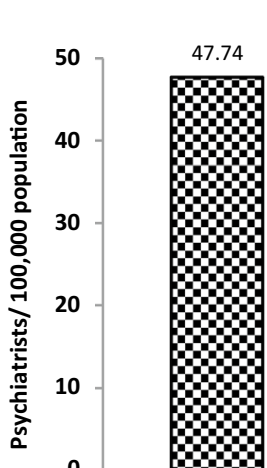

Child and adolescent psychiatrists among European countries by Human Development Index (HDI) rank

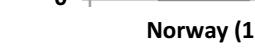

b

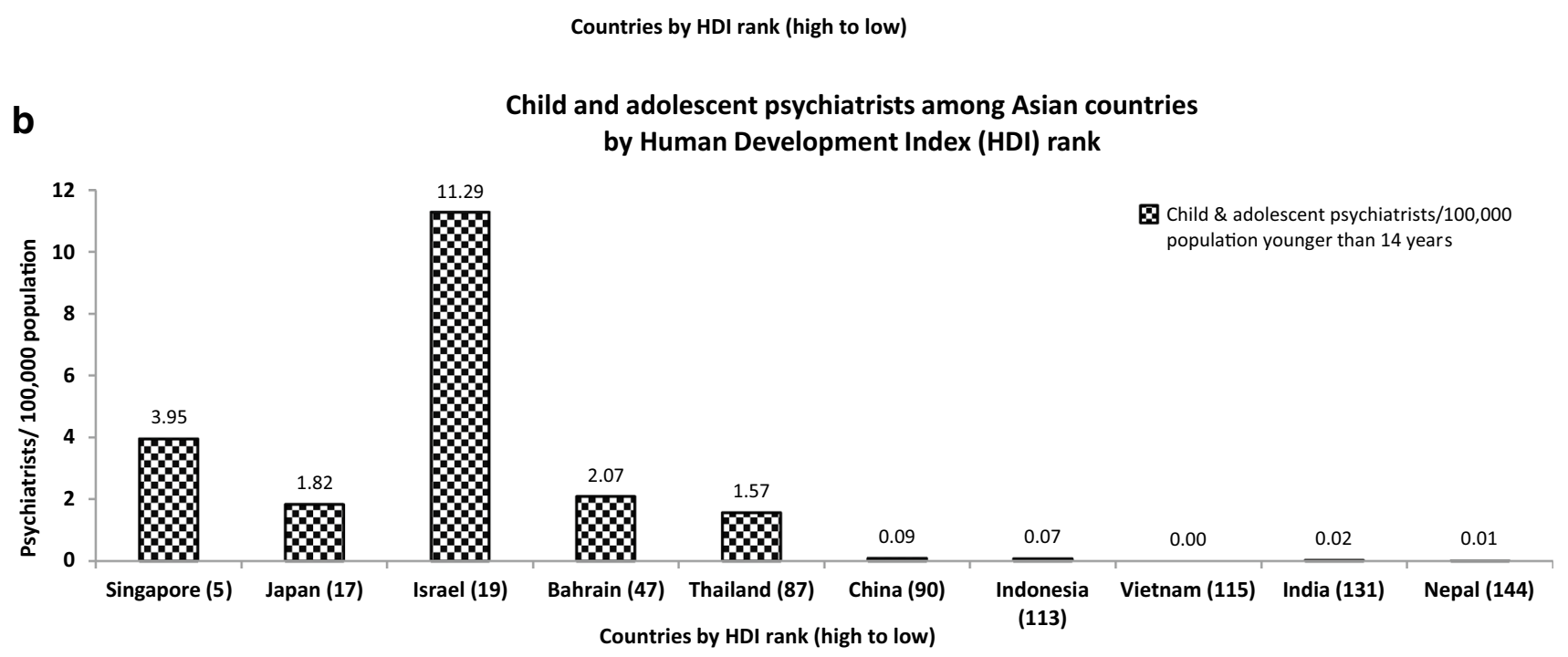

Child and adolescent psychiatrists among Asian countries by Human Development Index (HDI) rank
Child \& adolescent psychiatrists $/ 100,000$ population younger than 14 years
Fig. 1 a Child and adolescent psychiatrists among European countries per 100,000 population younger than 14 years by Human Development Index (HDI) rank (in parentheses). b Child and adolescent

schemes or out of pocket payments may to some extent act independently of the HDI. Finally, the data were based on information collected from a single or few CAP professionals in each country. The respondents have reported the most accurate information on CAP in each country from available sources (e.g., psychiatric associations, publications, etc.). However, possible differences in the accuracy of the available updated information need to be considered while interpreting the findings.

Our findings highlight the unmet needs in CAP in a large number of countries globally [5]. In addition to the impact of socioeconomic development and the HDI, possible effects of sociocultural influences and geographic distribution of the countries on the mental health workforce in CAP, particularly in Asia, need to be taken into account. When considering these findings, together with the fact that more than half of the children are currently living in Asia, our findings also highlight the enormity of the problem. Especially psychiatrists among Asian countries per 100,000 population younger than 14 years by Human Development Index (HDI) rank (in parentheses)

in low- and middle-income countries, there is an immense need for more specialized training in child and adolescent psychiatry.

Authors' contribution This is to verify that all the authors have approved this final version of the manuscript, approve of their authorship and agree to publication of this manuscript.

\section{References}

1. Costello EJ, Mustillo S, Keeler G, Angold A (2004) Prevalence of psychiatric disorders in childhood and adolescence. In: Levin BL, Petrila J, Hennessy KD (eds) Mental health services: a public health perspective, 2 nd edn. Oxford University Press, Oxford, pp $111-128$

2. Polanczyk GV, Salum GA, Sugaya LS, Caye A, Rohde LA (2015) Annual research review: a meta-analysis of the worldwide prevalence of mental disorders in children and adolescents. J Child Psychol Psychiatry 56(3):345-365 
3. World Health Organization. Adolescents: health risks and solutions. http://www.who.int/mediacentre/factsheets/fs345/en/. Accessed 2 Jan 2017

4. Mokdad AH, Forouzanfar MH, Daoud F, Mokdad AA, El Bcheraoui C, Moradi-Lakeh M, Kyu HH, Barber RM, Wagner J, Cercy K, Kravitz H, Coggeshall M, Chew A, O'Rourke KF, Steiner C, Tuffaha M, Charara R, Al-Ghamdi EA, Adi Y, Afifi RA, Alahmadi H, AlBuhairan F, Allen N, AlMazroa M, Al-Nehmi AA, AlRayess Z, Arora M, Azzopardi P, Barroso C, Basulaiman M, Bhutta ZA, Bonell C, Breinbauer C, Degenhardt L, Denno D, Fang J, Fatusi A, Feigl AB, Kakuma R, Karam N, Kennedy E, Khoja TA, Maalouf F, Obermeyer CM, Mattoo A, McGovern T, Memish ZA, Mensah GA, Patel V, Petroni S, Reavley N, Zertuche DR, Saeedi M, Santelli J, Sawyer SM, Ssewamala F, Taiwo K, Tantawy M, Viner RM, Waldfogel J, Zuñiga MP, Naghavi M, Wang H, Vos T, Lopez AD, Al Rabeeah AA, Patton GC, Murray CJ (2016) Global burden of diseases, injuries, and risk factors for young people's health during 1990-2013: a systematic analysis for the Global Burden of Disease Study 2013. Lancet 387(10036):2383-2401

5. World Health Organization (2005) Atlas: child and adolescent mental health resources. http://apps.who.int/iris/bitstream/1066 5/43307/1/9241563044_eng.pdf?ua=1. Accessed 3 Jan 2017

6. Morris J, Belfer M, Daniels A et al (2011) Treated prevalence of and mental health services received by children and adolescents in 42 low-and-middle-income countries. J Child Psychol Psychiatry 52(12):1239-1246
7. Sourander A, Chudal R (2017) The debate on child and adolescent psychiatric research is going global. Eur Child Adolesc Psychiatry 26(5):509-510

8. Chudal R (2016) Eurasia Child Mental Health Group Report. World Child and Adolescent Psychiatry 11:10-11. https://www. aacap.org/App_Themes/AACAP/docs/homepage/headlines/2017 /WORLD_CAP_Dec_2016.pdf. Accessed 8 Mar 2017

9. World Bank. Population data. http://data.worldbank.org/indicato r/SP.POP.TOTL. Accessed 10 Apr 2017

10. United Nations Development Programme (2016) Human Development Index. http://hdr.undp.org/en/content/human-developm ent-index-hdi. Accessed 3 May 2017

11. Leventhal. "Too Big to Fail? Too Small to Survive?. World child and adolescent Psychiatry. ISSUE 6, April 2014. http://www. wpanet.org/uploads/Sections/Child_and_Adolescent_Psychiat ry/WCAP\%202014\%20apr.pdf. Accessed 14 Mar 2017

12. Schulze TG, Treichel KC (2002) The European Federation of Psychiatric Trainees (EFPT) — an integral part of the European harmonisation of psychiatric education and practise. Eur Psychiatry 17(5):300-305

13. Hill P, Rothenberger A (2005) Can we-and should we-have a Europsychiatry for Children and Adolescents? The work of the UEMS Section and Board for Child and Adolescent Psychiatry/ Psychotherapy. Eur Child Adolesc Psychiatry 14(8):466-470 